\title{
Preparation and in vitro/ex vivo evaluation of nanoemulsion for transnasal delivery of paliperidone
}

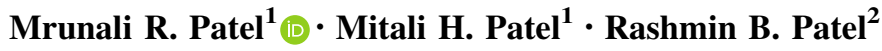

Received: 27 June 2015/Accepted: 8 March 2016/Published online: 29 March 2016

(c) The Author(s) 2016. This article is published with open access at Springerlink.com

\begin{abstract}
Paliperidone was formulated in mucoadhesive nanoemulsion with the aim of improving its solubility and transnasal delivery, which can further utilized for its preclinical evaluation. Solubility of Paliperidone in oils, emulsifiers, co-emulsifiers was determined to identify nanoemulsion components. Emulsifier and co-emulsifiers were screened for their ability to emulsify selected oily phase. Phase diagrams were constructed to identify the area of nanoemulsification. Paliperidone nanoemulsion was formulated using spontaneous nanoemulsification method. The three nanoemulsions (PMNE4, PMNE5, and PMNE6) containing 5.71-6.80\% oleic acid as an oily phase, $47.62-51.63 \%$ labrasol and plurol oleique CC 497 as emulsifier mixture (1:1), 42.86-45.58\% (wt/wt) aqueous phase having a suitable optical transparency of $98.33-99.33 \%$, globule size of $28.8-43.2 \mathrm{~nm}$ and polydispersity of $0.129-0.152$ were selected for the incorporation of mucoadhesive polymer. The PMNE6 showed highest flux $\left(5.072 \pm 0.13 \mu \mathrm{g} / \mathrm{cm}^{2} / \mathrm{min}\right)$ with enhancement ratio of 1.1 as compared to Paliperidone solution (PS). The diffusion co-efficient of PMNE6 was significantly higher than PMNE5, PMNE4 and PS and followed higuchi model. The formulation was found to be free from nasal cilio toxicity. All formulations were found to be stable for 6 months at room temperature.
\end{abstract}

Mrunali R. Patel

mrunalipatel@gmail.com

1 Department of Pharmaceutics and Pharmaceutical Technology, Indukaka Ipcowala College of Pharmacy, New Vallabh Vidyanagar, Gujarat 388121, India

2 Department of Pharmaceutical Chemistry and Quality Assurance, A. R. College of Pharmacy and G. H. Patel Institute of Pharmacy, Vallabh Vidynagar, Gujarat 388120, India
Keywords Ex vivo permeation study - Nanoemulsion . Nasal cilio toxicity study $\cdot$ Paliperidone

\section{Introduction}

Schizophrenia is one of the psychotic mental disorders and affects an individual's thoughts, behaviors, and social functioning. The major problem in treating mental disorders is due to the inability of the therapeutic agents to surpass the blood brain barrier (BBB) and the blood cerebrospinal fluid barrier. One of the possibilities to overcome this barrier is drug delivery to brain using novel drug delivery systems like nanoemulsions exploring nanotechnology (Canuso et al. 2008; Patel et al. 2010, 2013a , b, 2014).

Paliperidone (PALI) is an atypical antipsychotic agent that belongs to the class of benz-isoxazole and approved for the treatment of schizophrenia (Canuso et al. 2008; Patel et al. 2010, 2013a, b, 2014). It is a lipophilic drug having oral bioavailabity of $28 \%$. This is primarily attributed to its poor aqueous solubility, and high lipophilicity and extensive first pass metabolism. Considering the limitations, a strategy which will increase PALI solubility, reduce its first pass metabolism and overcome BBB through delivering PALI directly to the target site is highly desirable. The utility of nanoemulsions (NEs) has been successfully established in optimizing the therapeutic performance of many lipophilic drugs. Recently, increasing attention has been focused on nanoemulsion based drug delivery due to their ease of preparation with biocompatible formulation components, unique properties such as smaller droplet size $(50-200 \mathrm{~nm})$, increased drug solubility, and improved mucosal permeation, which further justifies rational of this study. 
An alternative route, intranasal drug delivery system has now been recognized as a reliable route for the administration of drugs next to parenteral and oral routes. The olfactory region of the nasal mucosa provides a direct connection between the nose and the brain. The advantages of nasal route are rapid absorption; higher bioavailability reduced first pass metabolism, non-invasive administration, and selfmedication as well as improved patient compliance (Canuso et al. 2008; Patel et al. 2013a, b, 2014). It is also reported in our earlier studies that absorption of PALI through transnasal route was more efficient when given in an oil based formulation (microemulsion) (Patel et al. 2013a, b). These studies outcomes render PALI as a potent candidate for lipid based systems such as nanoemulsion for intranasal delivery. However, these formulations may suffer from drawbacks such as rapid nasal mucocilliary clearance of PALI. To get the better of this, many research groups have demonstrated the use of a potent mucoadhesive system which improves the nasal residential time and prolongs the intimate contact of drug with nasal mucosa with improved absorption.

Taken together, this information has led our research group to a formulated mucoadhesive nanoemulsion of PALI with the aim of improving its solubility and transnasal delivery. This paper describes the formulation and in vitro/ex vivo evaluation of nanoemulsion based intranasal drug delivery system containing paliperidone.

\section{Materials and methods}

\section{Materials}

Paliperidone pure powder was obtained as a gift sample from Torrent pharmaceutical Ltd. (Ahmedabad, India). Labrafil M 1944, labrafac CC, labrasol, plurol oleique CC 497, and lauroglycol 90, (Gattefosse Saint-Priest, France) were procured as gratis samples. Polycarbophil AA-1, (pharmagrade, molecular weight approximately 3.5 million) was procured as gratis sample from Lubrizol Advanced Material India Pvt Ltd. (Mumbai, India). Oleic acid, tween 80, potassium dihydrogen phosphate, methanol and propylene glycol were purchased from Suvidhinath Laboratories (Vadodara, Gujarat). Double distilled water was used throughout the study. All other chemicals and solvents were of analytical reagent grade, and used as received without further purification.

\section{Paliperidone nanoemulsion development}

\section{Solubility study}

The solubility of PALI in various oils, emulsifiers, coemulsifiers was determined by a shake flask method. Briefly, an excess quantity of PALI was added in each stoppered glass vial containing $2 \mathrm{ml}$ of the selected oils, emulsifiers and co-emulsifiers, and mixed using cyclomixer. The vials were then incubated at $37 \pm 1.0{ }^{\circ} \mathrm{C}$ in an isothermal shaker for $72 \mathrm{~h}$. The samples were centrifuged at $3000 \mathrm{rpm}$ for $15 \mathrm{~min}$ and subsequently filtered through $0.22 \mu \mathrm{m}$ membrane filter. The filtrate was assayed by HPTLC method to estimate the amount of drug solubilized. All measurements were done in triplicate (Patel et al. 2010).

\section{Construction of phase diagram}

Phase diagram was constructed to define the extent and nature of the NE regions. Phase diagrams were constructed using spontaneous nanoemulsification method at an ambient temperature. For construction of phase diagram, emulsifier was blended with co-emulsifier in fixed weight ratios $(\mathrm{Km}, 1: 1,2: 1$ and 3:1 w/w). Each emulsifier and coemulsifier mixture (Emix) was then mixed with oil at ambient temperature. For each phase diagram, the ratio of oil to the Emix was varied as 9:1, 8:2, 7:3, 6:4, 5:5, 4:6, $3: 7,2: 8,1: 9(w / w)$. Water was added dropwise to each oilEmix mixture under vigorous stirring. After equilibrium, the samples were visually checked and determined as being clear/transparent (Elshafeey et al. 2009; Jogani et al. 2008; Kumar et al. 2008; Patel et al. 2009, 2013a, b).

NE which shows phase separation was rejected. The area of $\mathrm{NE}$ was identified for respective $\mathrm{Km}$ in which $\mathrm{NE}$ with desired globule size and stability were obtained.

\section{Preparation of nanoemulsion formulations}

The PALI loaded NE (PNE) were prepared by the dissolving PALI $(5 \mathrm{mg} / \mathrm{mL})$ in oil. Emulsifiers were weighed accurately and mixed with oil to form homogenous isotropic mixture. Required amount of water was added to the mixture to obtain PNE. PALI loaded mucoadhesive NE (PMNE) was prepared by the addition of polycarbophil AA-1 $(0.50 \%$, $\mathrm{w} / \mathrm{w})$ to PNE and the dispersion stirred for $1 \mathrm{~h}$. The composition of PNE and PMNE is shown in Table 1.

\section{Preparation of solution}

The PS $(5 \mathrm{mg} / \mathrm{mL})$ meant for the comparative evaluation was prepared by dissolving PALI $(50 \mathrm{mg})$ in $10 \mathrm{~mL}$ of propylene glycol (PG).

\section{Evaluation of nanoemulsion}

Globule size and zeta potential

The globule size and polydispersity index (PDI) of NE was measured by photon correlation spectroscopy (PCS) with 
Table 1 The composition of the PALI loaded nanoemulsion and mucoadhesive nanoemulsion formulations

\begin{tabular}{|c|c|c|c|c|c|c|c|c|c|c|}
\hline Ingredients (w/w) & PNE1 & PNE2 & PNE3 & $\mathrm{PNE}^{\mathrm{a}}$ & PNE5 $^{\mathrm{a}}$ & PNE6 $^{\mathrm{a}}$ & PNE7 & PNE8 & PNE9 & PNE10 \\
\hline Oleic acid & 2.38 & 2.08 & 1.85 & 1.70 & 1.56 & 1.43 & 1.75 & 2.05 & 2.4 & 2.3 \\
\hline Labrasol:plurol oleique cc 497 (3:1) & 9.52 & 10.41 & 11.11 & 11.90 & 12.5 & 12.86 & - & - & - & - \\
\hline Tween 80:propylene glycol (1:1) & - & - & - & - & - & - & 15.73 & 16.39 & 16.82 & 16.85 \\
\hline Distilled water & 13.10 & 12.5 & 12.04 & 11.39 & 10.93 & 10.71 & 7.52 & 6.56 & 5.77 & 5.34 \\
\hline
\end{tabular}

Each formulation contains paliperidone $5 \mathrm{mg} / \mathrm{mL}$

${ }^{a}$ PNE4, PNE5 and PNE 6 formulations additionally contain $0.5 \% \mathrm{wt} / \mathrm{wt}$ of polycarbophil as mucoadhesive agent, and further referred as PMNE4, PMNE5 and PMNE6 respectively

in-built Zetasizer (Nano ZS, Malvern Instruments, UK) at $633 \mathrm{~nm}$. Helium-neon gas laser having intensity of $4 \mathrm{~mW}$ was the light source. The globule size was calculated using Stokes-Einstein relationship by Zetasizer Software. Electrophoretic mobility $(\mu \mathrm{m} / \mathrm{s})$ was measured using small volume disposable zeta cell and converted to zeta potential by in-built software using Helmholtz-Smoluchowski equation (Elshafeey et al. 2009; Jogani et al. 2008; Kumar et al. 2008; Patel et al. 2009, 2013a, b).

\section{Viscosity}

The viscosity of NE was measured using a Brookfield Viscometer LVDV-IIIU (Brookfield Engineering LABS, Stoughton, MA) with spindle SC 18 at $8-30 \mathrm{rpm}$. All aspects of testing were controlled using optional Rheocalc Software (Patel et al. 2009).

\section{Drug content}

For determination of drug content, about $1 \mathrm{~mL}$ of the $\mathrm{NE}$ was taken in a $10 \mathrm{~mL}$ volumetric flask and dissolved in methanol. It was diluted appropriately and analyzed using reported HTPLC method (Patel et al. 2010).

Further, prepared nanoemulsion formulations were characterized by the measurement of $\mathrm{pH}$, conductivity, percentage transmittance, and refractive index (Patel et al. 2009).

\section{In vitro release studies}

In vitro release study was conducted using a diffusion cell (Orchid Scientifics, Nashik, India) with a diameter of $10 \mathrm{~mm}$. The receiver chamber containing $20 \mathrm{~mL}$ diffusion medium (phosphate buffer, $\mathrm{pH}$ 5.0) was kept at controlled experimental temperature $\left(37 \pm 0.5{ }^{\circ} \mathrm{C}\right)$. Diffusion medium was continuously stirred using teflon coated magnetic bar at a constant rate, in such a manner that surface of dialysis membrane (Hi-media) was just rinsed by diffusion medium. Samples from receiver compartment were withdrawn at predetermined time interval for $4 \mathrm{~h}$ and analyzed using HPTLC method (Patel et al. 2010).

\section{Ex vivo permeation study}

The freshly excised sheep nasal mucosa (from the slaughter house), except the septum part, was kept in phosphate buffer saline (PBS), pH 6.4 for $15 \mathrm{~min}$ to equilibrate. The excised superior nasal membrane was then mounted on the diffusion cell (diameter of $10 \mathrm{~mm}$ and mucosa thickness $0.2 \pm 0.1 \mathrm{~mm})$. The tissue was stabilized using phosphate buffer ( $\mathrm{pH} 5)$ in both the compartments and allowed to stir for 15 min on a magnetic stirrer (Kumar et al. 2009; Lalani et al. 2014; Patel et al. 2013a, b).

For ex vivo permeation study, each formulation $(1 \mathrm{~mL})$ was placed in the donor compartment of the diffusion cell. The temperature of the receiver chamber containing $20 \mathrm{~mL}$ of diffusion media (phosphate buffer, $\mathrm{pH}$ 5.0) was controlled at $37 \pm 0.5{ }^{\circ} \mathrm{C}$. Samples from receiver compartment were withdrawn at predetermined time interval for $4 \mathrm{~h}$ and analyzed using HPTLC method (Patel et al. 2010). Different evaluation parameters such as $\%$ drug diffused, flux, diffusion co-efficient and enhancement ratio $\left(E_{\mathrm{r}}=\right.$ PALI flux at steady state (Jss) of NE formulation/Jss of control vehicle) were derived. The permeation kinetic of PALI from each formulation was evaluated by fitting different kinetic models (Jogani et al. 2008; Kumar et al. 2008; Patel et al. 2009, 2013a, b).

\section{Nasal cilio toxicity study}

Histopathological studies were performed using sheep nasal mucosa similarly that of ex vivo diffusion study, to evaluate nasal toxicity of developed NE formulation. Three sheep nasal mucosa pieces (P1, P2, P3) with uniform thickness were selected and mounted on Franz diffusion cells. P1 was treated with $0.5 \mathrm{~mL}$ of PBS (pH 6.4, negative control), P2 with $0.5 \mathrm{~mL}$ of isopropyl alcohol (positive control), and P3 was treated with PMNE6 for $1 \mathrm{~h}$. After $1 \mathrm{~h}$, the mucosa were rinsed with PBS (pH 6.4) and subjected to histological studies and photographed by microscope (Polarizing Microscope RPL-55 Series, Radical Instruments, India) (Jogani et al. 2008; Kumar et al. 2008; Patel et al. 2009, 2013a, b). 


\section{Infrared study}

The infrared (IR) spectra (between 4000 and $400 \mathrm{~cm}^{-1}$ ) of PALI, plain NE, optimized PNE6 and PMNE6 were taken using an IR spectrophotometer (Spectrum GX FT-IR, Perkin Elmer, Norwalk, CT) to detect any possible interaction between the drug and excipients (Patel et al. 2009, 2015).

\section{Stability study}

Optimized formulations were subjected to stability studies for a period of 6 months at room temperature. After 6 months of storage, the NE formulations were subjected to test for physical stability via creaming, phase separation, or flocculation via accelerated centrifugation cycle $(3000 \times g$ for $15 \mathrm{~min})$ and chemical stability via $\mathrm{pH}$, drug content (Azeem et al. 2009; Borhade et al. 2012; Patel et al. 2009).

\section{Statistical analysis}

All experiments were performed in triplicates. All data are reported as mean \pm standard deviation (SD) and the groups were compared using ANOVA, with $p<0.05$ considered statistically significant.

\section{Results and discussion}

\section{Screening of nanoemulsion components}

The solubility of practically insoluble PALI was found highest in the oleic acid $(184.29 \pm 2.00 \mathrm{mg} / \mathrm{mL})$ and least in labrafil M $1944 \mathrm{CS}(3.14 \pm 0.46 \mathrm{mg} / \mathrm{mL})$. Further, it is reported in the literature that oleic acid act as a lipophilic permeation enhancer and can be useful to improve the membrane permeability (Azeem et al. 2009; Patel et al.
2015; Pierre et al. 2006). Hence, oleic acid was selected as the oily phase for the preparation of NE (Pierre et al. 2006).

An important criterion for selection of the emulsifiers is that the required hydrophilic lipophilic balance (HLB) value to form the oil-water NE be greater than 10. Emulsifiers play a major role in the formation and stabilization of NEs. They prevent the coalescence of drops by reducing the interfacial tension and by forming a stearic barrier against globule coalescence. PALI has got highest solubility in tween 80 (T-80) (HLB 15) and labrasol (HLB 14) as compared to other emulsifiers. Therefore, T- 80 and LAB were chosen as an emulsifier for the preparation of NEs (Azeem et al. 2009; Borhade et al. 2012; Patel et al. 2009).

Most single-chain emulsifiers do not lower the oil-water interfacial tension sufficiently to form NEs and short to medium chain length alcohols are necessary as co-emulsifiers. The co-emulsifier also ensures that the interfacial film is flexible enough to deform readily around each globule as their intercalation between the primary emulsifier molecules decreases both the polar head group interactions. Transient negative interfacial tension and fluid interfacial film are rarely achieved by the use of single emulsifier; usually, addition of a co-emulsifier is necessary. The presence of co-emulsifier decreases the bending stress of interface and allows the interfacial film sufficient flexibility to take up different curvatures required to form NEs over a wide range of composition. Thus, the co-emulsifiers selected for the study were PG and plurol oleiuqe CC 497 (PO-497) which has HLB value of 11.6 and 6, respectively.

\section{Pseudo ternary phase diagram}

A ternary phase diagram explains the selection of the formulations from the phase diagrams to avoid metastable formulations having minimum emulsifier concentration, in the least possible time. The pseudo ternary phase diagrams were constructed by varying T-80: PG and LAB: PO-497 ratios $(\mathrm{Km})$ as 1:1, 2:1 and 3:1 (Figs. 1, 2),

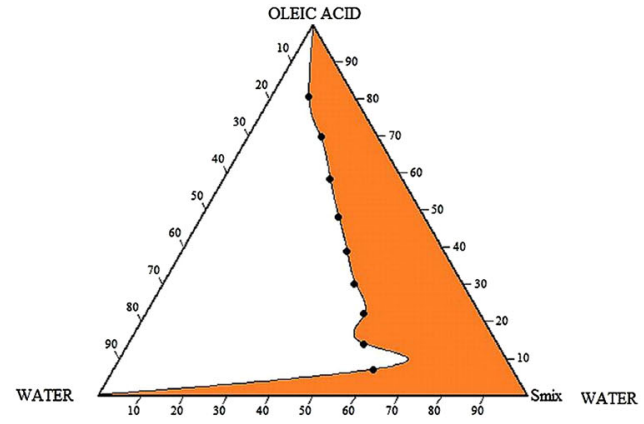

(A)

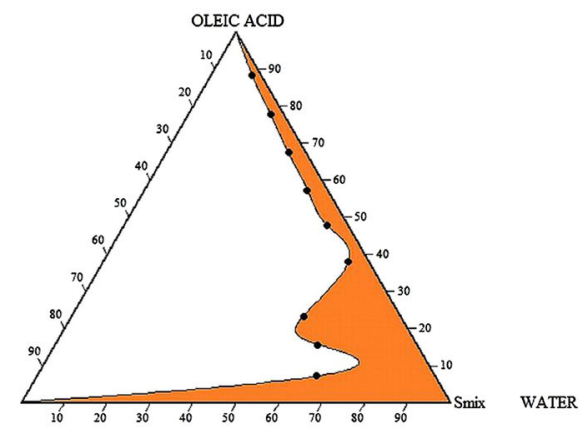

(B)

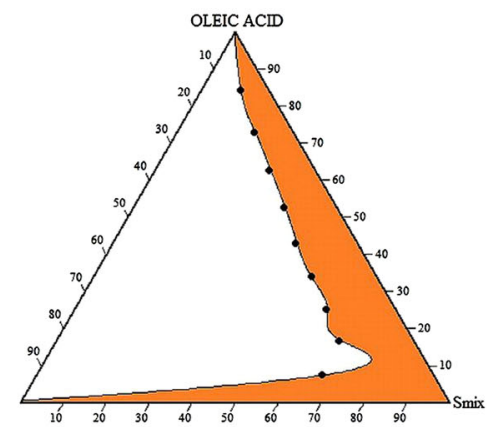

(C)

Fig. 1 The pseudo ternary phase diagrams of the oleic acid, tween 80: propylene glycol (Smix), water system at the 1:1 (a), 2:1 (b) and 3:1 (c) weight ratios of Smix $(\mathrm{Km})$ at ambient temperature. The dark area represents nanoemulsion region 


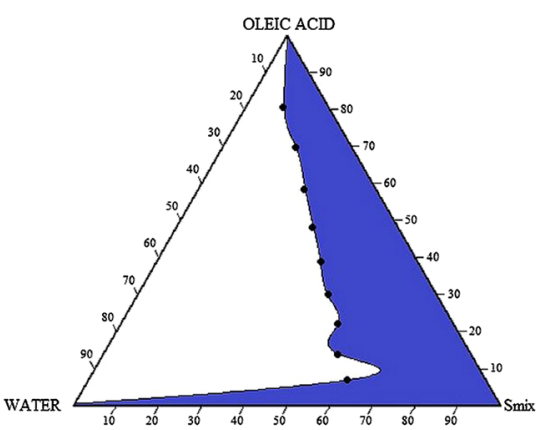

(A)

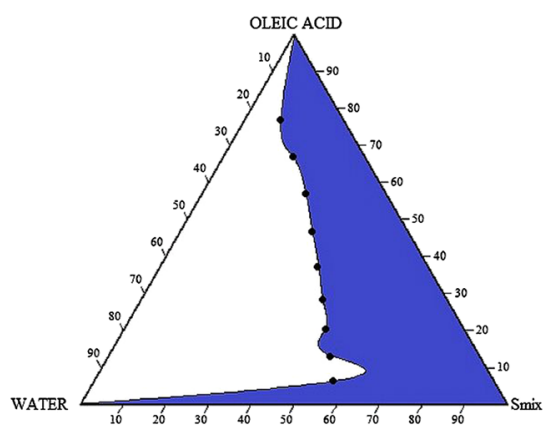

(B)

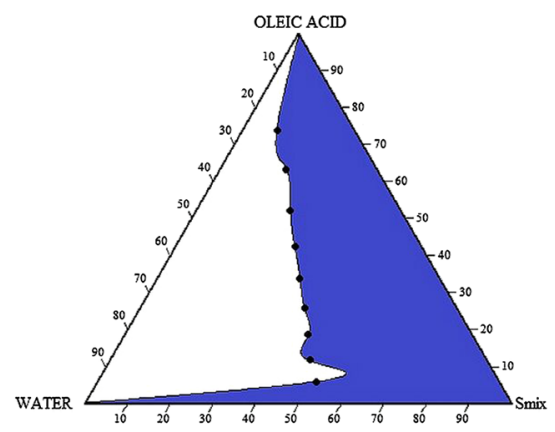

(C)

Fig. 2 The pseudo ternary phase diagrams of the oleic acid, labrasol: plurol oleique CC 497 (Smix), water system at the 1:1 (a), 2:1 (b) and 3:1 (c) weight ratios of Smix $(\mathrm{Km})$ at ambient temperature. The dark area represents nanoemulsion region

respectively. The shaded areas of phase diagrams show the NE regions, whereas the non-shaded area displays the multiphase emulsion region. The area of $\mathrm{NE}$ isotropic region changed slightly in size with $\mathrm{Km}$ in order of $3: 1>2: 1>1: 1$ for LAB:PO-497 and $1: 1>2: 1>3: 1$ for T-80:PG, respectively. It was observed that the highest NE area was obtained at Km-3 for LAB:PO-497 and Km-1 for T-80: PG at 1:1, respectively. Hence, these values were kept fixed for further studies.

\section{Physicochemical characterization of nanoemulsion}

The $\mathrm{pH}$ values of all the formulated NE were found within the physiological range (5-6.5), which is one of the formulation considerations that may help in reducing the irritation produced upon instillation. Conductivity measurements rely on the poor conductivity of oil compared with water and give low values in w/o emulsions, where oil is the continuous phase. The reverse happens for $\mathrm{o} / \mathrm{w}$ emulsion. The conductivity measurements (0.107$0.165 \mathrm{mS} / \mathrm{cm}$ ) indicate $\mathrm{NEs}$ to be of oil in water type. \% $T$ values of all NE formulations were between 97 and $100 \%$. Hence, all the NEs were found to be optically clear. Refractive index is the net value of the components of $\mathrm{NE}$ and indicates isotropic nature of formulation. The data in Table 2 indicate that the mean value of the refractive index for all the formulations was approximately similar. The results revealed that there were no interactions between $\mathrm{NE}$ components and drug. Viscosity of all formulated $\mathrm{NE}$ formulations was very low which is shown in Table 2. Low viscosity values ensure easy handling, packing and hasslefree administration of formulations (Kumar et al. 2009; Lalani et al. 2014; Patel et al. 2013a, b).

The globule size of all formulated NEs was found below $200 \mathrm{~nm}$. The results indicated that increasing the concentration of Emix, resulted in improved NE clarity and a smaller globule size. Such a decrease in globule size is the result of more emulsifier being available to stabilize the oil-water interface and due to the increase in emulsifier interfacial area and decrease in interfacial tension. Furthermore, the decrease in the globule size reflects the formation of a better close packed film of emulsifier at the oilwater interface, thereby stabilizing the oil globules. Polydispersity index (PDI) is a measure of globule homogeneity and it varies from 0.0 to 1.0. Polydispersity is the ratio of standard deviation to mean globule size; hence, it indicates the uniformity of globule size within the formulation (Kumar et al. 2009; Lalani et al. 2014; Patel et al. 2013a, b). The polydispersity index was found to be between 0.129 and 0.263 for formulations PNE1-PNE10 (Table 2).

Generally, an increase of electrostatic repulsive forces between $\mathrm{NE}$ globules prevents the coalescence of $\mathrm{NE}$ globules. On the contrary, a decrease of electrostatic repulsive forces will cause phase separation. Zeta potential gives certain information about the surface charge properties, and further the long-term physical stability of the NEs. As all the excipient used were nonionic in nature, low zeta potential values could be attributed to the drug molecules having ionizable group (Lalani et al. 2014; Patel et al. 2013a, b). The zeta potential values of all formulations are reported in Table 2.

PALI content of NE formulations was found in the range of $97.93-99.00 \% \mathrm{w} / \mathrm{w}$ of theoretical value $(5 \mathrm{mg} / \mathrm{mL})$ (Table 2).

The results of in vitro release study indicates that PNE6 showed highest cumulative percentage release $(85.09 \pm 0.78)$ and PNE10 showed the lowest cumulative percentage release value $(63.33 \pm 0.49)$ as depicted in Table 2, and was not statistically significant $(p>0.05)$. Also it was observed that the release rate of NE formulations containing $\mathrm{LAB}$ as emulsifier were higher as compared to NE formulations containing T-80 as emulsifier (Fig. 3). Therefore, the PNE4, PNE5, and PNE6 formulations having suitable physicochemical properties and 


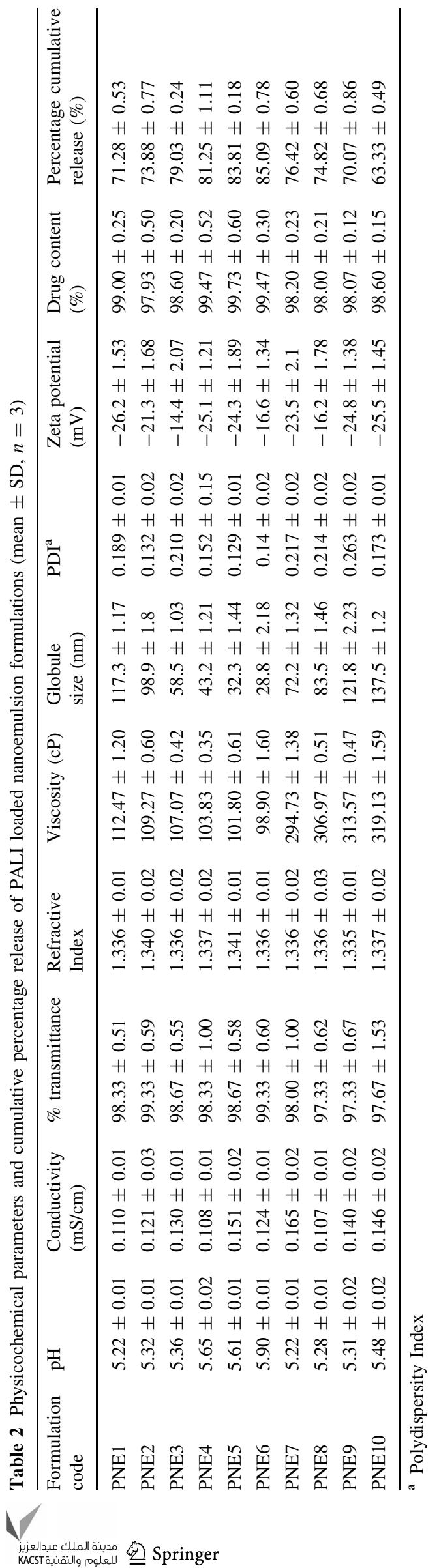

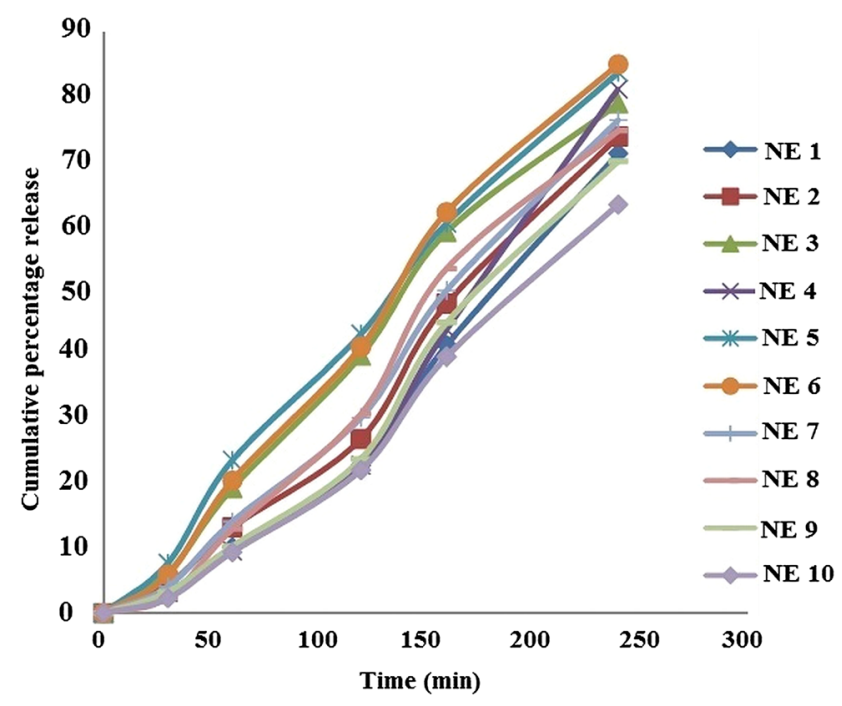

Fig. 3 Cumulative percentage release of nanoemulsion formulations NE1 to NE10

higher drug release rate were selected as optimized formulations for further studies (Patel et al. 2013a, b).

\section{Formulation and characterization of mucoadhesive nanoemulsion}

Polycarbophil has been studied extensively as mucoadhesive platform for drug delivery to the nasal mucosa (Kumar et al. 2009; Lalani et al. 2014; Patel et al. 2013a, b) in a concentration of $0.5 \% \mathrm{w} / \mathrm{w}$. This anionic polymer is most widely employed mucoadhesive polymer within pharmaceutical formulation due to its high mucoadhesive functionality and low toxicity. Polycarbophil form easily modified networks, is a non-irritant, non-toxic and considered safe for use by FDA. Thus, polycarbophil was selected as mucoadhesive polymer and incorporated in optimized NE formulations (PNE4, PNE5 and PNE6) at $0.5 \%$ w/v concentration (Barakat et al. 2006; Karasulu et al. 2008; Kumar et al. 2009) to obtain PALI loaded mucoadhesive NE formulations (PMNE4, PMNE5 and PMNE6). Characterization parameters for optimized PALI mucoadhesive NEs are shown in Table 3. All studied muucoahesive NE formulations showed suitable physicochemical parameters.

\section{Ex vivo permeation study}

In ex vivo diffusion studies of PALI formulations, successful diffusion through sheep nasal mucosa was obtained and the results are presented in Fig. 4, and the calculated diffusion coefficients are tabulated in Table 4 along with the regression coefficients $\left(r^{2}\right)$ for first-order, Higuchi, and zero-order modeling of the diffusion profiles for each formulation (Barakat et al. 2006). 
Table 3 Characterization parameters of PALI solution (PS) and optimized PALI mucoadhesive nanoemulsion (PMNE)

\begin{tabular}{|c|c|c|c|c|c|c|c|}
\hline $\begin{array}{l}\text { Formulation } \\
\text { code }\end{array}$ & $\mathrm{pH}$ & $\begin{array}{l}\text { Conductivity } \\
(\mathrm{mS} / \mathrm{cm})\end{array}$ & $\begin{array}{l}\text { Viscosity } \\
(\mathrm{cP})\end{array}$ & $\begin{array}{l}\text { Globule size } \\
(\mathrm{nm})\end{array}$ & $\mathrm{PDI}^{\mathrm{a}}$ & $\begin{array}{l}\text { Zeta potential } \\
(\mathrm{mV})\end{array}$ & $\begin{array}{l}\text { Drug content } \\
(\%)\end{array}$ \\
\hline PS & $5.51 \pm 0.03$ & - & - & - & - & - & $99.34 \pm 0.35$ \\
\hline PMNE4 & $5.61 \pm 0.02$ & $0.110 \pm 0.01$ & $124.27 \pm 1.10$ & $47.4 \pm 2.11$ & $0.210 \pm 0.01$ & $-31.3 \pm 1.54$ & $99.75 \pm 0.68$ \\
\hline PMNE5 & $5.68 \pm 0.02$ & $0.121 \pm 0.01$ & $119.47 \pm 0.65$ & $43.3 \pm 1.24$ & $0.143 \pm 0.02$ & $-32.9 \pm 1.70$ & $99.15 \pm 0.61$ \\
\hline PMNE6 & $5.97 \pm 0.02$ & $0.130 \pm 0.01$ & $116.73 \pm 0.95$ & $31.1 \pm 1.44$ & $0.178 \pm 0.04$ & $-29.7 \pm 1.14$ & $99.60 \pm 0.53$ \\
\hline
\end{tabular}

${ }^{a}$ Polydispersity Index

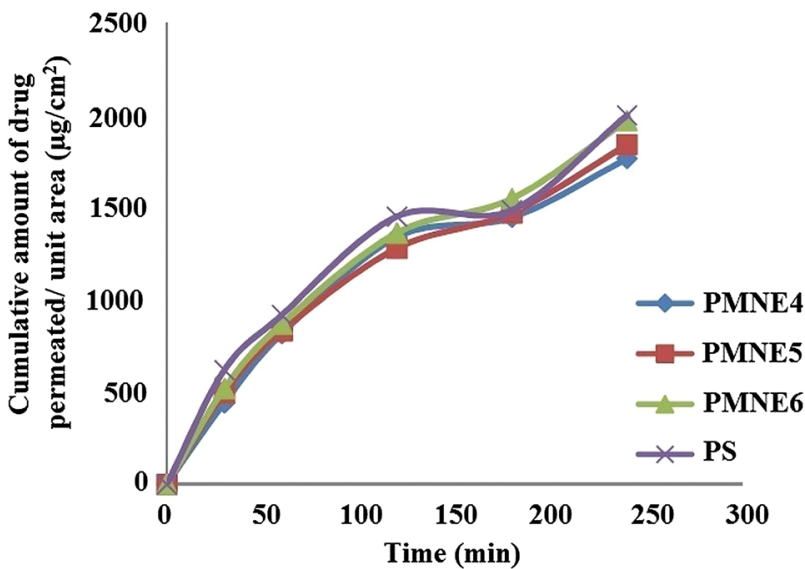

Fig. 4 Cumulative amount of drug permeated per unit area versus time profile of PS, PMNE4, PMNE5 and PMNE6

PALI show better diffusion from PMNE6 as compared to PMNE5, PMNE4 and PS through sheep nasal mucosa after $4 \mathrm{~h}(p<0.05)$. The decreasing order of diffusion coefficient for the tested formulations was PMNE4 $<$ PS $<$ PMNE5 < PMNE6. For PMNE6, the drug exhibited highest diffusion coefficient $\left(2.04 \times 10^{-5} \pm 0.022 \times\right.$ $\left.10^{-5} \mathrm{~cm}^{2} / \mathrm{min}\right)$ and flux $\left(5.072 \pm 0.13 \mu \mathrm{g} / \mathrm{cm}^{2} / \mathrm{min}\right)$ with enhancement ratio of $1: 1$ as compared to PS. The mucoadhesive NEs demonstrated higher diffusion due to the presence of mucoadhesive agent that probably due to its intrinsic character tends to adhere to mucosa, thereby causing increased contact time, and hence increased diffusion (Jogani et al. 2008; Kumar et al. 2008, 2009; Patel et al. 2009, 2013a, b).
On modeling, the diffusion of drug from PALI formulations demonstrated higher $r^{2}$ values for the Higuchi model as compared to zero- and first-order models. This may be due to the fact that the diffusion system used has a reservoir compartment (donor compartment) and sheep mucosa acts as a barrier or controlling membrane; hence, the diffusion process will mimic and shall be closer to reservoir system than zero-order (concentration independent) or first-order (concentration gradient) diffusion. Thus, on the basis of highest diffusion coefficient, PALI loaded PMNE6 was considered as optimized batch and further evaluated (Jogani et al. 2008; Kumar et al. 2008, 2009; Patel et al. 2009, 2013a, b).

\section{Nasal cilio toxicity}

The nasal cilio toxicity studies were carried out to evaluate any potential toxic effects of excipients used in the formulation on the nasal mucosa. The nasal mucosa treated with PBS (pH 6.4, negative control) showed no sign of inflammation, erosion and nasociliary damage (Fig. 5a), and the nasal membrane remained intact, whereas an extensive damage to nasal mucosa coupled with loss of nasal cilia (Fig. 5b) could be observed with positive control. However, with PALI loaded MNE (PMNE6), damage to nasal mucosa was not observed (Fig. 5c), which substantiated the safety of the excipients used in the formulation. Therefore, formulation components can be considered as biocompatible, and may not induce serious histological changes in the nasal mucosa upon prolong use

Table 4 Steady state flux, enhancement ratio, diffusion co-efficient and modeling parameters of PALI solution (PS) and paliperidone loaded mucoadhesive nanoemulsions (PMNE4, PMNE5 and PMNE6) (mean $\pm \mathrm{SD}, n=3$ )

\begin{tabular}{|c|c|c|c|c|c|c|}
\hline \multirow[t]{2}{*}{ Formulation } & \multirow{2}{*}{$\begin{array}{l}\text { Steady state flux (Jss) } \\
\left(\mu \mathrm{g} / \mathrm{cm}^{2} / \mathrm{min}\right)\end{array}$} & \multirow{2}{*}{$\begin{array}{l}\text { Enhancement ratio } \\
\left(E_{\mathrm{r}}\right)\end{array}$} & \multirow{2}{*}{$\begin{array}{l}\text { Diffusion co-efficient } \\
\left(\mathrm{cm}^{2} / \mathrm{min}\right)\end{array}$} & \multicolumn{3}{|c|}{ Modeling parameters } \\
\hline & & & & $\begin{array}{l}\text { Zero-order } \\
\left(r^{2}\right)\end{array}$ & $\begin{array}{l}\text { First-order } \\
\left(r^{2}\right)\end{array}$ & $\begin{array}{l}\text { Higuchi } \\
\left(r^{2}\right)\end{array}$ \\
\hline PS & $4.573 \pm 0.21$ & 1.00 & $1.84 \times 10^{-5} \pm 0.013 \times 10^{-5}$ & 0.9112 & 0.8826 & 0.9805 \\
\hline PMNE4 & $3.544 \pm 0.27$ & 0.77 & $1.42 \times 10^{-5} \pm 0.019 \times 10^{-5}$ & 0.9211 & 0.9718 & 0.9746 \\
\hline PMNE5 & $4.679 \pm 0.50$ & 1.03 & $1.89 \times 10^{-5} \pm 0.015 \times 10^{-5}$ & 0.9329 & 0.9634 & 0.9897 \\
\hline PMNE6 & $5.072 \pm 0.13$ & 1.10 & $2.04 \times 10^{-5} \pm 0.022 \times 10^{-5}$ & 0.9438 & 0.9387 & 0.9877 \\
\hline
\end{tabular}




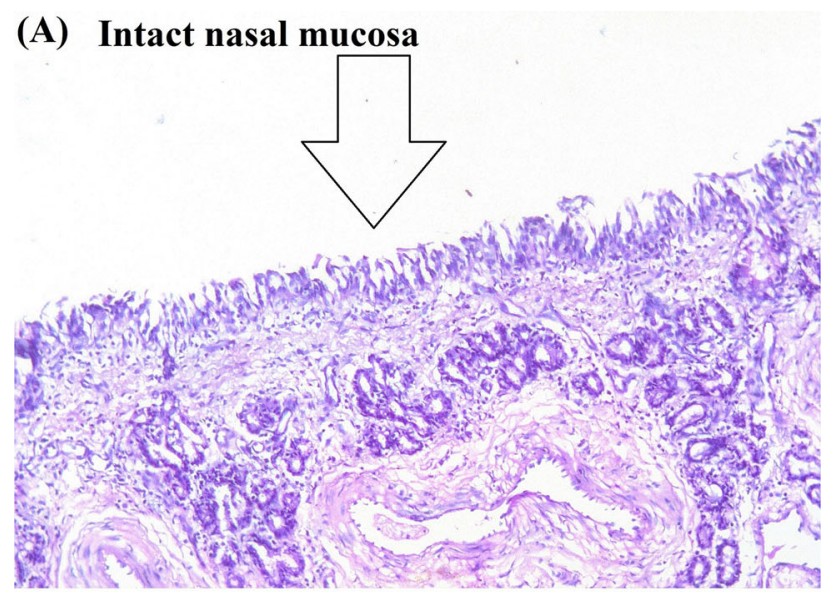

(B) Damaged nasal mucosa

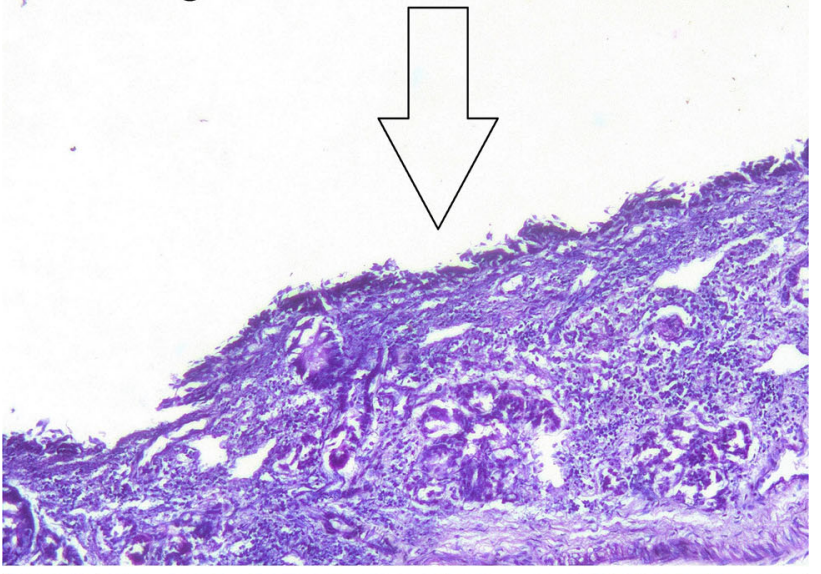

(C) Intact nasal mucosa

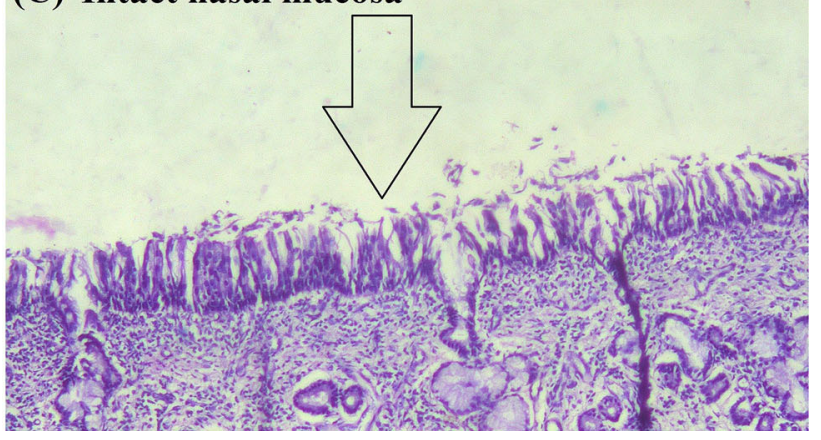

Fig. 5 Photographs of sheep nasal mucosa demonstrating histological characteristics when treated with (a) phosphate buffer saline $\mathrm{pH}$ 6.4 (b) isopropyl alcohol and (c) paliperidone loaded mucoadhesive nanoemulsion

(Barakat et al. 2006; Jadhav et al. 2011; Karasulu et al. 2008; Sharma et al. 2009).

\section{Infrared study}

The infrared spectra of PALI pure powder, plain NE, PNE6 and PMNE6 are as shown in the Fig. 6. PALI spectrum shows absorption bands at 2936 and $2758.6 \mathrm{~cm}^{-1}$ due to aliphatic $\mathrm{C}-\mathrm{H}$ stretching. A prominent band at $1655 \mathrm{~cm}^{-1}$ corresponds to $\mathrm{C}=\mathrm{O}$ stretching of the aryl acids and bands at 1536.6 and $1446 \mathrm{~cm}^{-1}$ related to $\mathrm{C}=\mathrm{C}$ and $\mathrm{C}=\mathrm{N}$ stretching. Bands at $1416 \mathrm{~cm}^{-1}$ are due to aliphatic $\mathrm{C}-\mathrm{H}$ bending and at $956.3,856.5$ and $817.6 \mathrm{~cm}^{-1}$ due to aromatic $\mathrm{C}-\mathrm{H}$ bending. Other bands at 1352.2 and $1122 \mathrm{~cm}^{-1}$ corresponds to $\mathrm{C}-\mathrm{N}$ stretching and $\mathrm{C}-\mathrm{F}$ stretching, respectively. The IR spectrum of PNE and PMNE is entirely different from PALI, while it closely resembles the spectrum of plain NE. The characteristic bands of PALI either have disappeared or few bands of PALI reduced in intensity probably due to the restriction inside the formulation matrix. The absorption band at $3400 \mathrm{~cm}^{-1}$ due to hydroxyl stretching was not affected in PNE and PMNE. Five different degradation products of PALI which shows IR absorption at $3399,3145,3057,3059,1721 \mathrm{~cm}^{-1}$ are reported in literature. PALI and PALI loaded formulations showed no degraded product peaks. Further, no interaction was observed among the excipients and drug in the studied formulations. Hence, it could be concluded that PALI was compatible with formulation components used (Patel et al. 2013a, b, 2015).

\section{Stability study}

In stability studies, the PNE showed no evidence of precipitation of drug, creaming, phase separation, and flocculation on visual observation and was found to be stable after centrifugation $(3000 \times g$ for $15 \mathrm{~min})$ at room temperature. The results of stability studies (Table 5) showed that there are negligible changes in the parameters such as drug content, $\%$ transmittance, of PALI loaded NE and PALI loaded MNE after 6 months of storage, thus substantiating the stability of formulation for 6 months (Barakat et al. 2006; Jadhav et al. 2011; Karasulu et al. 2008; Sharma et al. 2009).

\section{Conclusion}

NE formulation containing PALI was successfully developed using for the purpose of improving its solubility and transnasal delivery, which can be further utilized for its preclinical evaluation. Screening of formulation components helped to identify the most suitable excipients, whereas phase diagrams gave a good idea about the concentrations of the formulation components that should be used to achieve NE. Collectively, the results demonstrate that PMNE 6, due to its appropriate physicochemical properties, optimum emulsifier and co-emulsifier concentrations, highest diffusion coefficient, and free from nasal cilio toxicity may be more suitable for intranasal delivery 


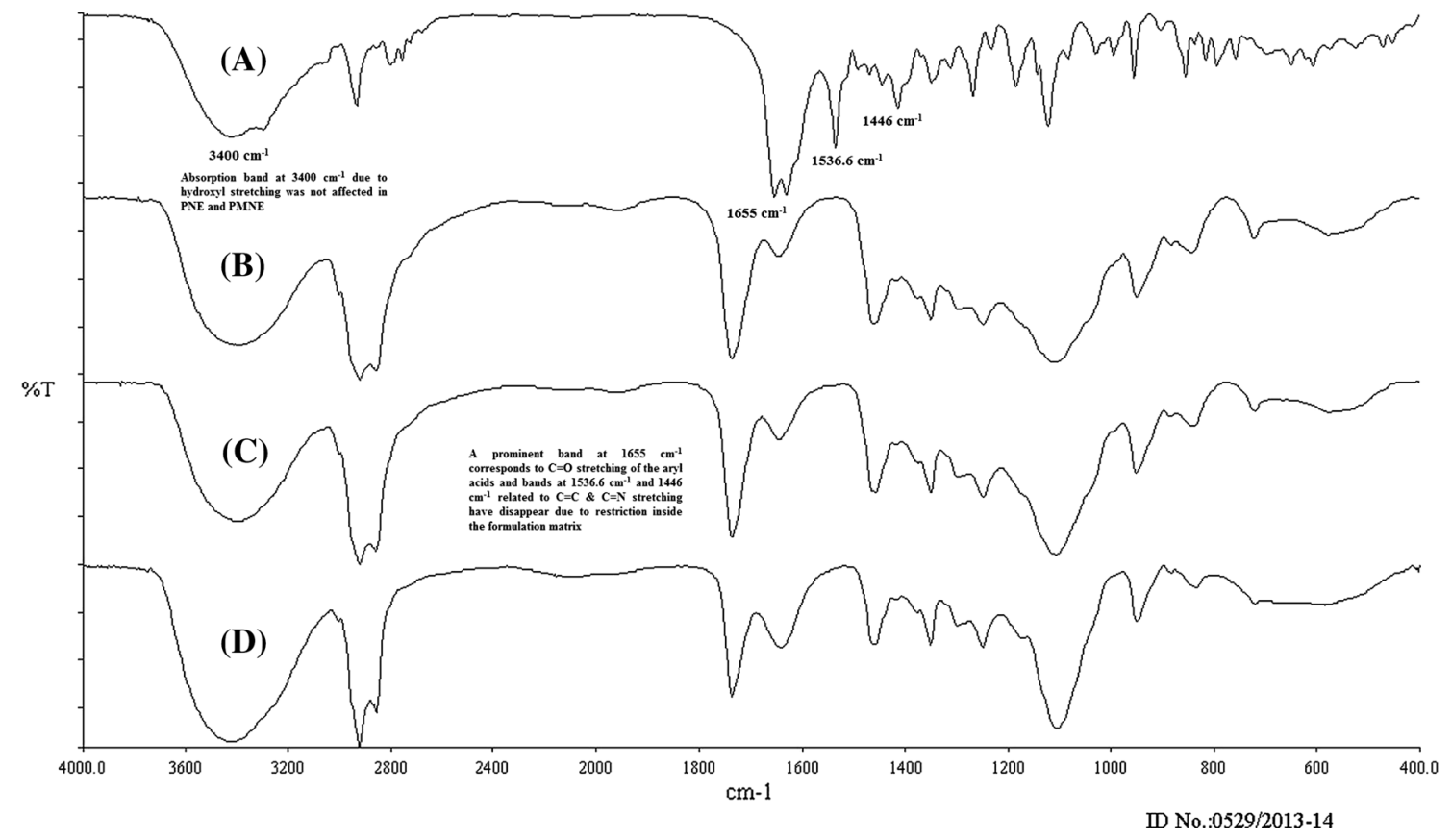

Fig. 6 Infra red spectra of $(a)$ paliperidone $(b)$ plain nanoemulsion $(c)$ paliperidone loaded nanoemulsion and $(d)$ paliperidone loaded mucoadhesive nanoemulsion

Table 5 Results of stability testing of the PALI nanoemulsion (PNE) and PALI mucoadhesive nanoemulsion containing $0.5 \%(\mathrm{w} / \mathrm{w})$ polycarbophil (PMNE) $(n=3)$ for 6 months

\begin{tabular}{lcl}
\hline Test & \multicolumn{1}{l}{ PNE6 } & PMNE6 \\
\hline \% Assay & $99.47 \pm 0.306$ & $99.6 \pm 0.529$ \\
$\%$ Transmittance & $99.33 \pm 0.58$ & - \\
Globule size $(\mathrm{nm})$ & $28.8 \pm 2.18$ & $31.1 \pm 1.44$ \\
Polydispersibility Index & $0.140 \pm 0.025$ & $0.178 \pm 0.035$ \\
Zeta potential $(\mathrm{mV})$ & $-16.6 \pm 1.34$ & $-29.7 \pm 1.14$
\end{tabular}

which could be developed as a novel regime for intranasal administration of PALI.

Acknowledgments Authors are thankful to Torrent Pharmaceutical Ltd. (India) for the gift sample of PALI pure powder, Sophisticated Instrumentation Center for Applied Research and Testing (SICART) (Vallabh Vidyanagar, India) for providing facilities for carrying out analytical work, Pathology Department (Veterinary College, Anand) for very kind help in toxicity study, Gattefosse (Saint-Priest, France), Abitec Corporation (Janesville, USA), Lubrizol Advance Material India Pvt. Ltd. (Mumbai, India), Noveon (Cleveland, USA) for providing gratis samples of excipients.

\section{Compliance with ethical standards}

Conflict of interest The author(s) confirm that this article content has no conflicts of interest.

Open Access This article is distributed under the terms of the Creative Commons Attribution 4.0 International License (http:// creativecommons.org/licenses/by/4.0/), which permits unrestricted use, distribution, and reproduction in any medium, provided you give appropriate credit to the original author(s) and the source, provide a link to the Creative Commons license, and indicate if changes were made.

\section{References}

Azeem A, Rizwan M, Ahmad FJ, Iqba Z, Khar RK, Aqil M, Talegaonkar S (2009) Nanoemulsion components screening and selection: a technical note. AAPS Pharm Sci Tech 10:69-76

Barakat NS, Omar SA, Ahmed AAE (2006) Carbamazepine uptake into rat brain following intra-olfactory transport. J Pharm Pharmacol 58:63-72

Borhade V, Pathak S, Sharma S, Patravale V (2012) Clotrimazole nanoemulsion for malaria chemotherapy. Part I: preformulation studies, formulation design and physicochemical evaluation. Int J Pharm 431:138-148

Canuso CM, Youssef EA, Bossie CA, Turkoz I, Schreiner A, Simpson GM (2008) Paliperidone extended-release tablets in schizophrenia patients previously treated with risperidone. Int Clin Psychopharm 23:209-215

Elshafeey AH, Bendas ER, Mohamed OH (2009) Intranasal microemulsion of sildenafil citrate: in vitro evaluation and in vivo pharmacokinetic study in rabbits. AAPS Pharm Sci Tech 10:361-364

Jadhav SA, Landge SB, Choudhari PM, Solanki PS, Bembalkar SR, Mathad VT (2011) Stress degradation behavior of paliperidone, an antipsychotic drug, and development of suitable stabilityindicating RP-LC method. Chromatogr Res Int 2011:1-10

Jogani VV, Shah PJ, Mishra P, Mishra AK, Misra AR (2008) Intranasal mucoadhesive microemulsion of tacrine to improve brain targeting. Alzheimer Dis Assoc Disord 22:116-124

Karasulu E, Yavasoglu A, Evrenşanal Z, Uyanıkgil Y, Karasulu HY (2008) Permeation studies and histological examination of sheep 
nasal mucosa following administration of different nasal formulations with or without absorption enhancers. Drug Del 15:219-225

Kumar M, Misra A, Mishra AK, Mishra P, Pathak K (2008) Mucoadhesive nanoemulsion-based intranasal drug delivery system of olanzapine for brain targeting. J Brain Targ $16: 806-814$

Kumar M, Pathak K, Misra A (2009) Formulation and characterization of nanoemulsion based drug delivery system of risperidone. Drug Dev Ind Pharm 35:387-395

Lalani J, Baradia D, Lalani R, Misra A (2014) Brain targeted intranasal delivery of tramadol: comparative study of microemulasion and nanoemulsion. Pharm Dev Tech. doi:10.3109/ 10837450.2014.959177

Patel MR, Patel RB, Parikh JR, Solanki AB, Patel BG (2009) Effect of formulation components on the in vitro permeation of microemulsion drug delivery system of fluconazole. AAPS Pharm Sci Tech 10:917-923

Patel RB, Patel MR, Bhatt KK, Patel BG (2010) HPTLC method development and validation: quantification of paliperidone in formulations and in vitro release study. Anal Methods 2:525-531
Patel RB, Patel MR, Bhatt KK, Patel BG (2013a) Paliperidone loaded mucoadhesive microemulsion in treatment of schizophrenia: formulation consideration. J Pharm Inn 8:195-204

Patel RB, Patel MR, Bhatt KK, Patel BG, Mishra A, Chutani K, Gaikwad RV, Samad A (2013b) Risperidone microemulsion for transnasal delivery: pharmacodynamic and pharmacokinetic evaluation. Pharm Nanotechnol 1:44-53

Patel MR, Patel RB, Bhatt KK, Patel BG, Gaikwad RV (2014) Paliperidone microemulsion for nose to brain targeted drug delivery system: pharmacodynamic and pharmacokinetic evaluation. Drug Deliv. doi:10.3109/10717544.2014.914602

Patel MR, Patel RB, Parikh JR, Patel BG (2015) Novel isotretinoin microemulsion based gel for targeted topical therapy of acne: formulation consideration, skin retention and skin irritation studies. Appl Nanosci. doi:10.1007/s13204-015-0457-z

Pierre MB, Ricci E, Tedesco AC, Bentley MV (2006) Oleic acid as optimizer of the skin delivery of 5-aminolevulinic acid in photodynamic therapy. Pharm Res 23:360-366

Sharma G, Mishra AN, Mishra P, Misra A (2009) Intranasal cabergoline: pharmacokinetic and pharmacodynamic studies. AAPS Pharm Sci Tech 10:1321-1330 